\title{
Linear and nonlinear convolution elliptic equations
}

\author{
Veli B Shakhmurov ${ }^{1,2}$ and Ismail Ekincioglu $3^{3 *}$
}

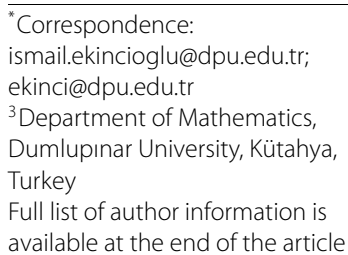

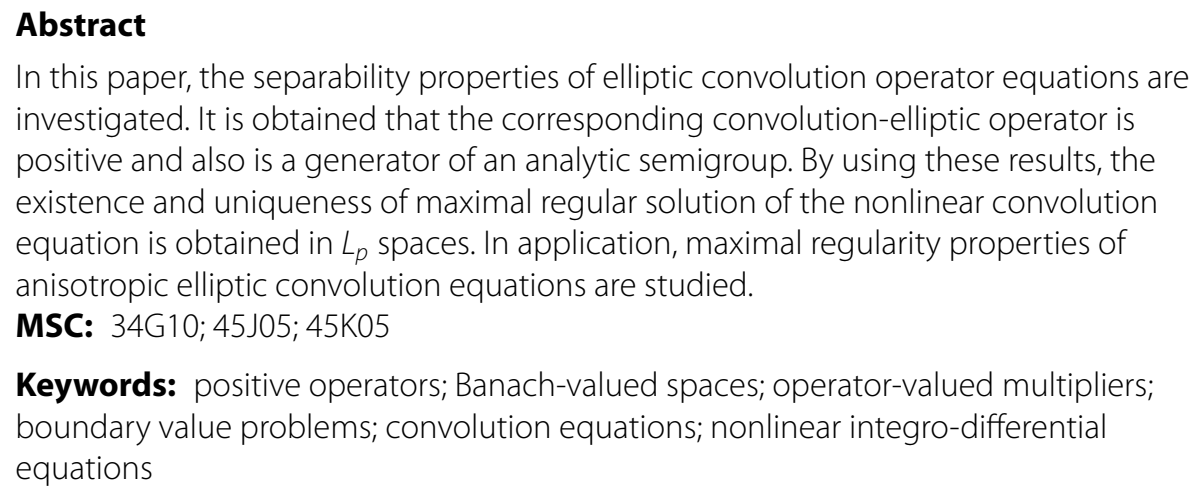

In this paper, the separability properties of elliptic convolution operator equations are investigated. It is obtained that the corresponding convolution-elliptic operator is positive and also is a generator of an analytic semigroup. By using these results, the existence and uniqueness of maximal regular solution of the nonlinear convolution equation is obtained in $L_{p}$ spaces. In application, maximal regularity properties of anisotropic elliptic convolution equations are studied.

MSC: $34 \mathrm{G} 10 ; 45 \mathrm{~J} 05 ; 45 \mathrm{~K} 05$

Keywords: positive operators; Banach-valued spaces; operator-valued multipliers; boundary value problems; convolution equations; nonlinear integro-differential equations

\section{Introduction}

In recent years, maximal regularity properties for differential operator equations, especially parabolic and elliptic-type, have been studied extensively, e.g., in [1-13] and the references therein (for comprehensive references, see [13]). Moreover, in [14, 15], on embedding theorems and maximal regular differential operator equations in Banach-valued function spaces have been studied. Also, in [16,17], on theorems on the multiplicators of Fourier integrals obtained, which were used in studying isotropic as well as anisotropic spaces of differentiable functions of many variables. In addition, multiplicators of Fourier integrals for the spaces of Banach valued functions were studied. On the basis of these results, embedding theorems are proved.

Moreover, convolution-differential equations (CDEs) have been treated, e.g., in [1, 1822] and [23]. Convolution operators in vector valued spaces are studied, e.g., in [24-26] and [27]. However, the convolution-differential operator equations (CDOEs) are a relatively less investigated subject (see [13]). The main aim of the present paper is to establish the separability properties of the linear $\mathrm{CDOE}$

$$
\sum_{|\alpha| \leq l} a_{\alpha} * D^{\alpha} u+(A+\lambda) * u=f(x)
$$

and the existence and uniqueness of the following nonlinear $\mathrm{CDOE}$

$$
\sum_{|\alpha| \leq l} a_{\alpha} * D^{\alpha} u+A * u=F\left(x, D^{\sigma} u\right)+f(x), \quad|\sigma| \leq l-1
$$

○ 2013 Shakhmurov and Ekincioglu; licensee Springer. This is an Open Access article distributed under the terms of the Creative Commons Attribution License (http://creativecommons.org/licenses/by/2.0), which permits unrestricted use, distribution, and reproduction in any medium, provided the original work is properly cited. 
in $E$-valued $L_{p}$ spaces, where $A=A(x)$ is a possible unbounded operator in a Banach space $E$, and $a_{\alpha}=a_{\alpha}(x)$ are complex-valued functions, and $\lambda$ is a complex parameter. We prove that the problem (1.1) has a unique solution $u$, and the following coercive uniform estimate holds

$$
\sum_{|\alpha| \leq l}|\lambda|^{1-\frac{|\alpha|}{l}}\left\|a_{\alpha} * D^{\alpha} u\right\|_{L_{p}\left(\mathbb{R}^{n} ; E\right)}+\|A * u\|_{L_{p}\left(\mathbb{R}^{n} ; E\right)}+|\lambda|\|u\|_{L_{p}\left(\mathbb{R}^{n} ; E\right)} \leq C\|f\|_{L_{p}\left(\mathbb{R}^{n} ; E\right)}
$$

for all $f \in L_{p}\left(\mathbb{R}^{n} ; E\right), p \in(1, \infty)$ and $\lambda \in S_{\varphi}$. The methods are based on operator-valued multiplier theorems, theory of elliptic operators, vector-valued convolution integrals, operator theory and etc. Maximal regularity properties for parabolic CDEs with bounded operator coefficients were investigated in [1].

\section{Notations and background}

Let $L_{p}(\Omega ; E)$ denote the space of all strongly measurable $E$-valued functions that are defined on the measurable subset $\Omega \subset \mathbb{R}^{n}$ with the norm

$$
\begin{aligned}
& \|f\|_{L_{p}(\Omega ; E)}=\left(\int\|f(x)\|_{E}^{p} d x\right)^{\frac{1}{p}}, \quad 1 \leq p<\infty, \\
& \|f\|_{L_{\infty}(\Omega ; E)}=\operatorname{ess} \sup _{x \in \Omega}\left[\|f(x)\|_{E}\right], \quad x=\left(x_{1}, x_{2}, \ldots, x_{n}\right) .
\end{aligned}
$$

Let $\mathbf{C}$ be the set of complex numbers, and let

$$
S_{\varphi}=\{\lambda ;|\lambda \in \mathbf{C},| \arg \lambda \mid \leq \varphi\} \cup\{0\}, \quad 0 \leq \varphi<\pi .
$$

A linear operator $A=A(x), x \in \Omega$ is said to be uniformly positive in a Banach space $E$ if $D(A(x))$ is dense in $E$, does not depend on $x$, and there is a positive constant $M$ so that

$$
\left\|(A(x)+\lambda I)^{-1}\right\|_{B(E)} \leq M(1+|\lambda|)^{-1}
$$

for every $x \in \Omega$ and $\lambda \in S_{\varphi}, \varphi \in[0, \pi)$, where $I$ is an identity operator in $E$, and $B(E)$ is the space of all bounded linear operators in $E$, equipped with the usual uniform operator topology. Sometimes, instead of $A+\lambda I$, we write $A+\lambda$ and denote it by $A_{\lambda}$. It is known (see [28], \$1.14.1) that there exist fractional powers $A^{\theta}$ of the positive operator $A$. Let $E\left(A^{\theta}\right)$ denote the space $D\left(A^{\theta}\right)$ with the graphical norm

$$
\|u\|_{E\left(A^{\theta}\right)}=\left(\|u\|^{p}+\left\|A^{\theta} u\right\|^{p}\right)^{\frac{1}{p}}, \quad 1 \leq p<\infty,-\infty<\theta<\infty .
$$

Let $S\left(\mathbb{R}^{n} ; E\right)$ denote Schwartz class, i.e., the space of $E$-valued rapidly decreasing smooth functions on $\mathbb{R}^{n}$, equipped with its usual topology generated by semi-norms. $S\left(\mathbb{R}^{n} ; C\right)$ denoted by just $S$. Let $S^{\prime}\left(\mathbb{R}^{n} ; E\right)$ denote the space of all continuous linear operators $L: S \rightarrow E$, equipped with the bounded convergence topology. Recall $S\left(\mathbb{R}^{n} ; E\right)$ is norm dense in $L_{p}\left(\mathbb{R}^{n} ; E\right)$ when $1 \leq p<\infty$. 
Let $\alpha=\left(\alpha_{1}, \alpha_{2}, \ldots, \alpha_{n}\right)$, where $\alpha_{i}$ are integers. An $E$-valued generalized function $D^{\alpha} f$ is called a generalized derivative in the sense of Schwartz distributions of the function $f \in$ $S^{\prime}\left(\mathbb{R}^{n}, E\right)$ if the equality

$$
\left(D^{\alpha} f\right)(\varphi)=(-1)^{|\alpha|} f\left(D^{\alpha} \varphi\right)
$$

holds for all $\varphi \in S$.

Let $F$ denote the Fourier transform. Through this section, the Fourier transformation of a function $f$ will be denoted by $\hat{f}$. It is known that

$$
F\left(D_{x}^{\alpha} f\right)=\left(i \xi_{1}\right)^{\alpha_{1}} \cdots\left(i \xi_{n}\right)^{\alpha_{n}} \hat{f}, \quad D_{\xi}^{\alpha}(F(f))=F\left[\left(-i x_{n}\right)^{\alpha_{1}} \cdots\left(-i x_{n}\right)^{\alpha_{n}} f\right]
$$

for all $f \in S^{\prime}\left(\mathbb{R}^{n} ; E\right)$.

Let $\Omega$ be a domain in $\mathbb{R}^{n}$. $C(\Omega ; E)$ and $C^{(m)}(\Omega ; E)$ will denote the spaces of $E$-valued bounded uniformly strongly continuous and $m$-times continuously differentiable functions on $\Omega$, respectively. For $E=\mathbf{C}$ the space $C^{(m)}(\Omega ; E)$ will be denoted by $C^{(m)}(\Omega)$. Suppose $E_{1}$ and $E_{2}$ are two Banach spaces. A function $\Psi \in L_{\infty}\left(\mathbb{R}^{n} ; B\left(E_{1}, E_{2}\right)\right)$ is called a multiplier from $L_{p}\left(\mathbb{R}^{n} ; E_{1}\right)$ to $L_{p}\left(\mathbb{R}^{n} ; E_{2}\right)$ if the map $u \rightarrow T u=F^{-1} \Psi(\xi) F u, u \in S\left(\mathbb{R}^{n} ; E_{1}\right)$ is well defined and extends to a bounded linear operator

$$
T: L_{p}\left(\mathbb{R}^{n} ; E_{1}\right) \rightarrow L_{p}\left(\mathbb{R}^{n} ; E_{2}\right)
$$

Let $Q$ denotes a set of some parameters. Let $\Phi_{h}=\left\{\Psi_{h} \in M_{p}^{p}\left(E_{1}, E_{2}\right), h \in Q\right\}$ be a collection of multipliers in $M_{p}^{p}\left(E_{1}, E_{2}\right)$. We say that $W_{h}$ is a collection of uniformly bounded multipliers (UBM) if there exists a positive constant $M$ independent on $h \in Q$ such that

$$
\left\|F^{-1} \Psi_{h} F u\right\|_{L_{p}\left(\mathbb{R}^{n} E_{2}\right)} \leq M\|u\|_{L_{p}\left(\mathbb{R}^{n} ; E_{1}\right)}
$$

for all $h \in Q$ and $u \in S\left(\mathbb{R}^{n} ; E_{1}\right)$.

A Banach space $E$ is called an $U M D$-space $[29,30]$ if the Hilbert operator

$$
(H f)(x)=\lim _{\varepsilon \rightarrow 0} \int_{\{|x-y|>\varepsilon\}} \frac{f(y)}{x-y} d y
$$

is bounded in $L_{p}(R, E), p \in(1, \infty)$ [29]. The $U M D$ spaces include, e.g., $L_{p}, l_{p}$ spaces and Lorentz spaces $L_{p q}, p, q \in(1, \infty)$.

A set $W \subset B\left(E_{1}, E_{2}\right)$ is called $R$-bounded (see $\left.[5,6,12]\right)$ if there is a positive constant $C$ such that

$$
\int_{0}^{1}\left\|\sum_{j=1}^{m} r_{j}(y) T_{j} u_{j}\right\|_{E_{2}} d y \leq C \int_{0}^{1}\left\|\sum_{j=1}^{m} r_{j}(y) u_{j}\right\|_{E_{1}} d y
$$

for all $T_{1}, T_{2}, \ldots, T_{m} \in W$ and $u_{1}, u_{2}, \ldots, u_{m} \in E_{1}, m \in N$, where $\left\{r_{j}\right\}$ is a sequence of independent symmetric $\{-1,1\}$-valued random variables on $[0,1]$. The smallest $C$, for which the above estimate holds, is called an $R$-bound of the collection $W$ and denoted by $R(W)$. 
A set $W_{h} \subset B\left(E_{1}, E_{2}\right)$, dependent on parameters $h \in Q$, is called uniformly $R$-bounded with respect to $h$ if there is a positive constant $C$, independent of $h \in Q$, such that for all $T_{1}(h), T_{2}(h), \ldots, T_{m}(h) \in W_{h}$ and $u_{1}, u_{2}, \ldots, u_{m} \in E_{1}, m \in \mathbf{N}$

$$
\int_{0}^{1}\left\|\sum_{j=1}^{m} r_{j}(y) T_{j}(h) u_{j}\right\|_{E_{2}} d y \leq C \int_{0}^{1}\left\|\sum_{j=1}^{m} r_{j}(y) u_{j}\right\|_{E_{1}} d y .
$$

This implies that $\sup _{h \in Q} R\left(W_{h}\right) \leq C$.

Definition 2.1 A Banach space $E$ is said to be a space, satisfying the multiplier condition, if for any $\Psi \in C^{(n)}\left(\mathbb{R}^{n} \backslash\{0\} ; B(E)\right)$ the $R$-boundedness of the set

$$
\left\{|\xi|^{|\beta|} D_{\xi}^{\beta} \Psi(\xi): \xi \in \mathbb{R}^{n} \backslash 0, \beta=\left(\beta_{1}, \beta_{2}, \ldots, \beta_{n}\right), \beta_{k} \in\{0,1\}\right\}
$$

implies that $\Psi$ is a Fourier multiplier, i.e., $\Psi \in M_{p}^{p}(E)$ for any $p \in(1, \infty)$.

The uniform $R$-boundedness of the set

$$
\left\{|\xi|^{|\beta|} D^{\beta} \Psi_{h}(\xi): \xi \in \mathbb{R}^{n} \backslash\{0\}, \beta \in\{0,1\}\right\}
$$

i.e.,

$$
\sup _{h \in Q} R\left(\left\{|\xi|^{|\beta|} D^{\beta} \Psi_{h}(\xi): \xi \in \mathbb{R}^{n} \backslash 0, \beta_{k} \in\{0,1\}\right\}\right) \leq C
$$

implies that $\Psi_{h}$ is a uniformly bounded collection of Fourier multipliers (UBM) in $L_{p}\left(\mathbb{R}^{n} ; E\right)$.

Remark 2.2 Note that if $E$ is $U M D$ space, then by virtue of $[5,7,12,25]$, it satisfies the multiplier condition. The $U M D$ spaces satisfy the uniform multiplier condition (see Proposition 2.4).

Definition 2.3 A positive operator $A$ is said to be a uniformly $R$-positive in a Banach space $E$ if there exists $\varphi \in[0, \pi)$ such that the set

$$
L_{A}=\left\{\xi(A+\xi)^{-1}: \xi \in S_{\varphi}\right\}
$$

is uniformly $R$-bounded.

Note that every norm bounded set in Hilbert spaces is $R$-bounded. Therefore, all sectorial operators in Hilbert spaces are $R$-positive.

Let $h \in R, m \in N$ and $e_{k}, k=1,2, \ldots, n$ be standard unit vectors of $\mathbb{R}^{n}$,

$$
\Delta_{k}(h) f(x)=f\left(x+h e_{k}\right)-f(x),
$$

and let $A=A(x), x \in \mathbb{R}^{n}$ be a closed linear operator in $E$ with domain $D(A)$ independent of $x$. The Fourier transformation of $A(x)$ is a linear operator with the same domain $D(A)$ 
defined as

$$
\hat{A} u(\varphi)=A u(\hat{\varphi}) \quad \text { for } u \in S^{\prime}\left(\mathbb{R}^{n} ; E(A)\right), \varphi \in S\left(\mathbb{R}^{n}\right) .
$$

(For details see [2, p.7].) Let $A=A(x)$ be a closed linear operator in $E$ with domain $D(A)$ independent of $x$. Then, it is differentiable if there is the limit

$$
\left(\frac{\partial A}{\partial x_{k}}\right) u=\lim _{h \rightarrow 0} \frac{\Delta_{k}(h) A(x) u}{h}, \quad k=1,2, \ldots, n, u \in D(A)
$$

in the sense of $E$-norm.

Let $A=A(x), x \in \mathbb{R}^{n}$ be closed linear operator in $E$ with domain $D(A)$ independent of $x$ and $u \in S^{\prime}\left(\mathbb{R}^{n}, E\right)$. We can define the convolution $A * u$ in the distribution sense by

$$
A * u(x)=\int_{\mathbb{R}^{n}} A(x-y) u(y) d y=\int_{\mathbb{R}^{n}} A(y) u(x-y) d y
$$

(see [2]).

Let $E_{0}$ and $E$ be two Banach spaces, where $E_{0}$ is continuously and densely embedded into $E$. Let $l$ be a integer number. $W_{p}^{l}\left(\mathbb{R}^{n} ; E_{0}, E\right)$ denote the space of all functions from $S^{\prime}\left(\mathbb{R}^{n} ; E_{0}\right)$ such that $u \in L_{p}\left(\mathbb{R}^{n} ; E_{0}\right)$ and the generalized derivatives $D_{k}^{l} u \in L_{p}\left(\mathbb{R}^{n} ; E\right)$ with the following norm

$$
\|u\|_{W_{p}^{l}\left(\mathbb{R}^{n} ; E_{0}, E\right)}=\|u\|_{L_{p}\left(\mathbb{R}^{n} ; E_{0}\right)}+\sum_{k=1}^{n}\left\|D_{k}^{l} u\right\|_{L_{p}\left(\mathbb{R}^{n} ; E\right)}<\infty
$$

It is clearly seen that

$$
W_{p}^{l}\left(\mathbb{R}^{n} ; E_{0}, E\right)=W_{p}^{l}\left(\mathbb{R}^{n} ; E\right) \cap L_{p}\left(\mathbb{R}^{n} ; E_{0}\right) .
$$

A function $u \in W_{p}^{l}\left(\mathbb{R}^{n} ; E(A), E\right)$ satisfying the equation (1.1) a.e. on $\mathbb{R}^{n}$, is called a solution of equation (1.1).

The elliptic CDOE (1.1) is said to be separable in $L_{p}\left(\mathbb{R}^{n} ; E\right)$ if for $f \in L_{p}\left(\mathbb{R}^{n} ; E\right)$ the equation (1.1) has a unique solution $u$, and the following coercive estimate holds

$$
\sum_{|\alpha| \leq l}\left\|a_{\alpha} * D^{\alpha} u\right\|_{L_{p}\left(\mathbb{R}^{n} ; E\right)}+\|A * u\|_{L_{p}\left(\mathbb{R}^{n} ; E\right)} \leq C\|f\|_{L_{p}\left(\mathbb{R}^{n} ; E\right)}
$$

where the constant $C$ do not depend on $f$.

In a similar way as Theorem $A_{0}$ in [31], Theorem $A_{0}$ and by reasoning as Theorem 3.7 in [7], we obtain the following.

Proposition 2.4 Let E be UMD space, $\Psi_{h} \in C^{n}\left(\mathbb{R}^{n} \backslash\{0\} ; B(E)\right)$ and suppose there is a positive constant $K$ such that

$$
\sup _{h \in Q} R\left(\left\{|\xi|^{|\beta|} D^{\beta} \Psi_{h}(\xi): \xi \in \mathbb{R}^{n} \backslash\{0\}, \beta_{k} \in\{0,1\}\right\}\right) \leq K .
$$

Then $\Psi_{h}$ is UBM in $L_{p}\left(\mathbb{R}^{n} ; E\right)$ for $p \in(1, \infty)$. 
Proof Really, some steps of proof trivially work for the parameter dependent case (see [7]). Other steps can be easily shown by setting

$$
\phi_{h}=\left\{|\xi|^{|\beta|} D^{\beta} \Psi_{h}(\xi): \xi \in \mathbb{R}^{n} \backslash\{0\}, \beta_{k} \in\{0,1\}\right\}
$$

instead of

$$
\left\{|\xi|^{|\beta|} D^{\beta} \Psi(\xi): \xi \in \mathbb{R}^{n} \backslash\{0\}, \beta_{k} \in\{0,1\}\right\}
$$

and by using uniformly $R$-boundedness of set $\phi_{h}$. However, parameter depended analog of Proposition 3.4 in [7] is not straightforward. Let $M_{h}$ and $M_{h, N} \in L_{1}^{\text {loc }}\left(\mathbb{R}^{n}, B(E)\right)$ be Fourier multipliers in $L_{p}\left(\mathbb{R}^{n} ; E\right)$. Let $M_{h, N}$ converge to $M_{h}$ in $L_{1}^{\text {loc }}\left(\mathbb{R}^{n}, B(E)\right)$, and let $T_{h, N}=F^{-1} M_{h, N} F$ be uniformly bounded with respect to $h$ and $N$. Then by reasoning as Proposition 3.4 in [7], we obtain that the operator function $T_{h}=F^{-1} M_{h} F=\lim _{N \rightarrow \infty} F^{-1} M_{h, N} F$ is uniformly bounded with respect to $h$. Hence, by using steps above, in a similar way as Theorem 3.7 in [7], we obtain the assertion.

Let $E_{1}$ and $E_{2}$ be two Banach spaces. Suppose that $T \in B\left(E_{1}, E_{2}\right)$ and $1 \leq p<\infty$. Then $\tilde{T} \in B\left(L_{p}\left(\mathbb{R}^{n} ; E_{1}\right), L_{p}\left(\mathbb{R}^{n} ; E_{2}\right)\right)$ will denote operator $(\tilde{T} f)(x)=T(f(x))$ for $f \in L_{p}\left(\mathbb{R}^{n} ; E_{1}\right)$ and $x \in \mathbb{R}^{n}$.

In a similar way as Proposition 2.11 in [12], we have

Proposition 2.5 Let $1 \leq p<\infty$. If $W \subset B\left(E_{1}, E_{2}\right)$ is $R$-bounded, then the collection $\tilde{W}=$ $\{\tilde{T}: T \in W\} \subset B\left(L_{p}\left(\mathbb{R}^{n} ; E_{1}\right), L_{p}\left(\mathbb{R}^{n} ; E_{2}\right)\right)$ is also $R$-bounded.

From [11], we obtain the following.

\section{Theorem 2.6 Let the following conditions be satisfied}

1. E is a Banach space satisfying the uniform multiplier condition, $p \in(1, \infty)$ and $0<h \leq h_{0}<\infty$ are certain parameters;

2. lis a positive integer, and $\alpha=\left(\alpha_{1}, \alpha_{2}, \ldots, \alpha_{n}\right)$ are $n$-tuples of nonnegative integer numbers such that $\varkappa=\frac{|\alpha|}{l}<1,0 \leq \mu<1-\varkappa$;

3. $A$ is an R-positive operator in $E$ with $0 \leq \varphi<\pi$.

Then the embedding $D^{\alpha} W_{p}^{l}\left(\mathbb{R}^{n} ; E(A), E\right) \subset L_{p}\left(\mathbb{R}^{n} ; E\left(A^{1-\varkappa-\mu}\right)\right)$ is continuous, and there exists a positive constant $C_{\mu}$ such that

$$
\left\|D^{\alpha} u\right\|_{L_{p}\left(\mathbb{R}^{n} ; E\left(A^{1-\varkappa-\mu))}\right.\right.} \leq C_{\mu}\left[h^{\mu}\|u\|_{W_{p}^{l}\left(\mathbb{R}^{n} ; E(A), E\right)}+h^{-(1-\mu)}\|u\|_{L_{p}\left(\mathbb{R}^{n} ; E\right)}\right]
$$

\section{Theorem 2.7 Let the following conditions be satisfied}

1. E is a Banach space satisfying the uniform multiplier condition, $p \in(1, \infty)$ and $0<h \leq h_{0}<\infty$ are certain parameters;

2. lis a positive integer, and $\alpha=\left(\alpha_{1}, \alpha_{2}, \ldots, \alpha_{n}\right)$ are $n$-tuples of nonnegative integer numbers such that $\varkappa=\frac{p|\alpha|+n}{p l}<1,0 \leq \mu<1-\varkappa$;

3. A is an R-positive operator in $E$ with $0 \leq \varphi<\pi$. 
Then the embedding $D^{\alpha} W_{p}^{l}\left(\mathbb{R}^{n} ; E(A), E\right) \subset C\left(\mathbb{R}^{n} ; E\left(A^{1-\varkappa-\mu}\right)\right)$ is continuous, and there exists a positive constant $C_{\mu}$ such that

$$
\left\|D^{\alpha} u\right\|_{C\left(\mathbb{R}^{n} ; E\left(A^{1-\varkappa-\mu}\right)\right)} \leq C_{\mu}\left[h^{\mu}\|u\|_{W_{p}^{l}\left(\mathbb{R}^{n} ; E(A), E\right)}+h^{-(1-\mu)}\|u\|_{L_{p}\left(\mathbb{R}^{n} ; E\right)}\right]
$$

for all $u \in W_{p}^{l}\left(\mathbb{R}^{n} ; E(A), E\right)$.

\section{Elliptic CDOE}

Condition 3.1 Assume that $a_{\alpha} \in L_{\infty}\left(\mathbb{R}^{n}\right)$ and the following hold

$$
L(\xi)=\sum_{|\alpha| \leq l} a_{\alpha}(\xi)(i \xi)^{\alpha} \in S_{\varphi_{1}}, \quad|L(\xi)| \geq C \sum_{k=1}^{n}\left|a_{k}\right|\left|\xi_{k}\right|^{l}
$$

where $\varphi_{1} \in[0, \pi), \xi=\left(\xi_{1}, \xi_{2}, \ldots, \xi_{n}\right) \in \mathbb{R}^{n}$.

In the following, we denote the operator functions by $\sigma_{i}(\xi, \lambda)$ for $i=0,1,2$.

Lemma 3.2 Assume Condition 3.1 holds, and $A(\xi)$ is a uniformly $\varphi$-positive operator in $E$ with $0 \leq \varphi<\pi-\varphi_{1}$. Then, the following operator functions

$$
\begin{aligned}
& \sigma_{0}(\xi, \lambda)=\lambda D(\xi, \lambda), \quad \sigma_{1}(\xi, \lambda)=A(\xi) D(\xi, \lambda) \\
& \sigma_{2}(\xi, \lambda)=\sum_{|\alpha| \leq l}|\lambda|^{1-\frac{|\alpha|}{l}} a_{\alpha}(\xi)(i \xi)^{\alpha} D(\xi, \lambda)
\end{aligned}
$$

are uniformly bounded, where $D(\xi, \lambda)=[A(\xi)+L(\xi)+\lambda]^{-1}$.

Proof By virtue of Lemma 2.3 in [4] for $L(\xi) \in S_{\varphi_{1}}, \lambda \in S_{\varphi}$ and $\varphi_{1}+\varphi<\pi$ there is a positive constant $C$ such that

$$
|\lambda+L(\xi)| \geq C(|\lambda|+|L(\xi)|)
$$

Since $L(\xi) \in S_{\varphi_{1}}$, in view of (3.1) and resolvent properties of positive operators, we get that $A(\xi)+L(\xi)+\lambda$ is invertible and

$$
\begin{aligned}
\left\|\sigma_{0}(\xi, \lambda)\right\|_{B(E)} & \leq M|\lambda|[1+|\lambda|+|L(\xi)|]^{-1} \leq M_{0}, \\
\left\|\sigma_{1}(\xi, \lambda)\right\|_{B(E)} & =\|I-(\lambda+L(\xi)) D(\xi, \lambda)\|_{B(E)} \\
& \leq 1+M|\lambda+L(\xi)|(1+|\lambda+L(\xi)|)^{-1} \leq M_{1} .
\end{aligned}
$$

Next, let us consider $\sigma_{2}$. It is clearly seen that

$$
\left\|\sigma_{2}(\xi, \lambda)\right\|_{B(E)} \leq C \sum_{|\alpha| \leq l}|\lambda|\left[|\xi||\lambda|^{-\frac{1}{l}}\right]^{|\alpha|}\|D(\xi, \lambda)\|_{B(E)} .
$$

Since $A$ is uniformly $\varphi$-positive and $L(\xi) \in S_{\varphi_{1}}$, then setting $y_{k}=\left(|\lambda|^{-\frac{1}{l}}\left|\xi_{k}\right|\right)^{\alpha_{k}}$ in the following well-known inequality

$$
y_{1}^{\alpha_{1}} y_{2}^{\alpha_{2}} \cdots y_{n}^{\alpha_{n}} \leq C\left(1+\sum_{k=1}^{n} y_{k}^{l}\right), \quad y_{k} \geq 0,|\alpha| \leq l,
$$


we obtain

$$
\left\|\sigma_{2}(\xi, \lambda)\right\|_{B(E)} \leq C \sum_{|\alpha| \leq l}|\lambda|\left[1+\sum_{k=1}^{n}\left|\xi_{k}\right|^{l}|\lambda|^{-1}\right][1+|\lambda+L(\xi)|]^{-1} .
$$

Taking into account the Condition 3.1 and (3.1)-(3.3), we get

$$
\left\|\sigma_{2}(\xi, \lambda)\right\|_{B(E)} \leq C\left(|\lambda|+\sum_{k=1}^{n}\left|\xi_{k}\right|^{l}\right)[1+|\lambda|+|L(\xi)|]^{-1} \leq C .
$$

Lemma 3.3 Assume Condition 3.1 holds, and $a_{\alpha} \in C^{(n)}\left(\mathbb{R}^{n}\right)$. Let $A(\xi)$ be a uniformly $\varphi$ positive operator in a Banach space $E$ with $0 \leq \varphi<\pi-\varphi_{1},\left[D^{\beta} A(\xi)\right] A^{-1}(\xi) \in C\left(\mathbb{R}^{n} ; B(E)\right)$ and let

$$
\begin{aligned}
& |\xi|^{\beta}\left|D^{\beta} a_{\alpha}(\xi)\right| \leq C_{1}, \quad \beta_{k} \in\{0,1\}, \xi \in \mathbb{R}^{n} \backslash\{0\}, 0 \leq|\beta| \leq n, \\
& \left\||\xi|^{\beta}\left[D^{\beta} A(\xi)\right] A^{-1}(\xi)\right\|_{B(E)} \leq C_{2}, \quad \beta_{k} \in\{0,1\}, \xi \in \mathbb{R}^{n} \backslash\{0\} .
\end{aligned}
$$

Then, operator functions $|\xi|^{\beta} D^{\beta} \sigma_{i}(\xi, \lambda)$ are uniformly bounded.

Proof Let us first prove that $\xi_{k} \frac{\partial \sigma_{1}}{\partial \xi_{k}}$ is uniformly bounded. Really,

$$
\left\|\xi_{k} \frac{\partial \sigma_{1}}{\partial \xi_{k}}\right\|_{B(E)} \leq\left\|I_{1}\right\|_{B(E)}+\left\|I_{2}\right\|_{B(E)}+\left\|I_{3}\right\|_{B(E)}
$$

where

$$
I_{1}=\left[\xi_{k} \frac{\partial A(\xi)}{\partial \xi_{k}}\right] D(\xi, \lambda), \quad I_{2}=A(\xi)\left[\xi_{k} \frac{\partial A(\xi)}{\partial \xi_{k}}\right][D(\xi, \lambda)]^{2}
$$

and

$$
I_{3}=A(\xi)\left[\xi_{k} \frac{\partial L(\xi)}{\partial \xi_{k}}\right] D^{2}(\xi, \lambda) .
$$

By using (3.1) and (3.5), we get

$$
\left\|I_{1}\right\|_{B(E)} \leq\left\|\left[\xi_{k} \frac{\partial A(\xi)}{\partial \xi_{k}}\right] A^{-1}(\xi)\right\|_{B(E)}\left\|\sigma_{1}\right\|_{B(E)} \leq C
$$

Due to positivity of $A$, by using (3.1) and (3.5), we obtain

$$
\left\|I_{2}\right\|_{B(E)} \leq\left\|\left[\xi_{k} \frac{\partial A(\xi)}{\partial \xi_{k}}\right] A^{-1}(\xi)\right\|_{B(E)}\left\|\sigma_{1}\right\|_{B(E)}^{2} \leq C .
$$

Since, $A(\xi)$ is uniformly $\varphi$-positive, by using (3.1), (3.3) and (3.4) for $\lambda \in S(\varphi)$ and $\varphi_{1}+\varphi<\pi$, we get

$$
\left\|I_{3}\right\|_{B(E)} \leq\left|\xi_{k} \frac{\partial L}{\partial \xi_{k}}\right|\|D(\xi, \lambda)\|_{B(E)}\left\|\sigma_{1}(\xi, \lambda)\right\|_{B(E)} \leq C .
$$


In a similar way, the uniform boundedness of $\sigma_{0}(\xi, \lambda)$ is proved. Next, we shall prove $\xi_{k} \frac{\partial \sigma_{2}}{\partial \xi_{k}}$ is uniformly bounded. Similarly,

$$
\left\|\xi_{k} \frac{\partial \sigma_{2}}{\partial \xi_{k}}\right\|_{B(E)} \leq\left\|J_{1}\right\|_{B(E)}+\left\|J_{2}\right\|_{B(E)}
$$

where

$$
\begin{aligned}
& J_{1}=\sum_{|\alpha| \leq l}|\lambda|^{1-\frac{|\alpha|}{l}}\left(\xi_{k} \frac{\partial a_{\alpha}}{\partial \xi_{k}}\right)\left[(i \xi)^{\alpha}+a_{\alpha}(\xi) i \alpha_{k}(i \xi)^{\alpha}\right] D(\xi, \lambda) \\
& J_{2}=\sum_{|\alpha| \leq l}|\lambda|^{1-\frac{|\alpha|}{l}} a_{\alpha}(\xi)(i \xi)^{\alpha}\left[\xi_{k} \frac{\partial a_{\alpha}}{\partial \xi_{k}}+a_{\alpha}(\xi)(i \xi)^{\alpha}+\xi_{k} \frac{\partial A(\xi)}{\partial \xi_{k}}\right][D(\xi, \lambda)]^{2} .
\end{aligned}
$$

Let us first show that $J_{1}$ is uniformly bounded. It is clear that

$$
\left\|J_{1}\right\|_{B(E)} \leq \sum_{|\alpha| \leq l}\left|\xi_{k} \frac{\partial a_{\alpha}}{\partial \xi_{k}}\right|\left\|\xi^{\alpha}|\lambda|^{1-\frac{|\alpha|}{l}} D(\xi, \lambda)\right\|_{B(E)} .
$$

Due to positivity of $A$, by virtue of (3.1) and (3.3)-(3.5), we obtain $\left\|J_{1}\right\|_{B(E)} \leq C$. In a similar way, we have $\left\|J_{2}\right\|_{B(E)} \leq C$. Hence, operator functions $\xi_{k} \frac{\partial \sigma_{i}}{\partial \xi_{k}}, i=0,1,2$ are uniformly bounded. From the representations of $\sigma_{i}(\xi, \lambda)$, it easy to see that operator functions $|\xi|^{\beta} D^{\beta} \sigma_{i}(\xi, \lambda)$ contain similar terms as $I_{k}$, namely, the functions $|\xi|^{\beta} D^{\beta} \sigma_{i}(\xi, \lambda)$ will be represented as combinations of principal terms

$$
\begin{aligned}
& \xi^{\sigma}\left[D_{\xi}^{\gamma} A(\xi)+D_{\xi}^{\gamma} a_{\alpha}(\xi)\right][D(\xi, \lambda)]^{|\beta|}, \\
& \sum_{|\alpha| \leq l}|\lambda|^{1-\frac{|\alpha|}{l}} \xi^{\sigma} D_{\xi}^{\gamma}\left[A(\xi)+a_{\alpha}(\xi)\right][D(\xi, \lambda)]^{|\beta|},
\end{aligned}
$$

where $|\sigma|+|\gamma| \leq|\beta|$. Therefore, by using similar arguments as above and in view of (3.6), one can easily check that

$$
|\xi|^{\beta}\left\|D^{\beta} \sigma_{i}(\xi, \lambda)\right\| \leq C, \quad i=0,1,2 .
$$

Lemma 3.4 Let all conditions of the Lemma 3.2 hold. Suppose that $E$ is a Banach space satisfying the uniform multiplier condition, and $A(\xi)$ is a uniformly $R$ positive operator in $E$. Then, the following sets

$$
\begin{aligned}
& S_{0}(\xi, \lambda)=\left\{|\xi|^{\beta} D_{\xi}^{\beta} \sigma_{0}(\xi, \lambda) ; \xi \in \mathbb{R}^{n} \backslash\{0\}\right\}, \\
& S_{1}(\xi, \lambda)=\left\{|\xi|^{\beta} D_{\xi}^{\beta} \sigma_{1}(\xi, \lambda) ; \xi \in \mathbb{R}^{n} \backslash\{0\}\right\}, \\
& S_{2}(\xi, \lambda)=\left\{|\xi|^{\beta} D_{\xi}^{\beta} \sigma_{2}(\xi, \lambda) ; \xi \in \mathbb{R}^{n} \backslash\{0\}\right\}
\end{aligned}
$$

are uniformly $R$-bounded for $\beta_{k} \in\{0,1\}$ and $0 \leq|\beta| \leq n$.

Proof Due to $R$-positivity of $A$ we obtain that the set

$$
B_{1}(\xi, \lambda)=\left\{[\lambda+L(\xi)] D(\xi, \lambda) ; \xi \in \mathbb{R}^{n} \backslash\{0\}\right\}
$$


is $R$ bounded. Since

$$
I-\sigma(\xi, \lambda)=A D(\xi, \lambda), \quad \sigma(\xi, \lambda)=[\lambda+L(\xi)] D(\xi, \lambda),
$$

the set $B_{2}(\xi, \lambda)=\left\{A D(\xi, \lambda) ; \xi \in \mathbb{R}^{n} \backslash\{0\}\right\}$ is $R$-bounded. Moreover, in view of Condition 3.1 and (3.1), there is a positive constant $M$ such that

$$
|\lambda||\lambda+L(\xi)|^{-1} \leq M
$$

Then, by virtue of Kahane's contraction principle, Lemma 3.5 in [5], we obtain that the set $B_{3}(\xi, \lambda)=\left\{\lambda D(\xi, \lambda) ; \xi \in \mathbb{R}^{n} \backslash\{0\}\right\}$ is uniformly $R$-bounded. Then by Lemma 3.2, we obtain the uniform $R$-boundedness of sets $B_{k}(\xi, \lambda)$, i.e,

$$
\sup _{\lambda} R\left\{B_{k}(\xi, \lambda)\right\} \leq M_{k}, \quad k=1,2,3 .
$$

Moreover, due to boundedness of $a_{\alpha}(\xi)$, in view of Condition 3.1 and by virtue of (3.1) and (3.3), we obtain

$$
\left.\left|\sum_{|\alpha| \leq l}\right| \lambda\right|^{1-\frac{|\alpha|}{l}} a_{\alpha}(\xi)(i \xi)^{\alpha} \mid \leq C_{1}(1+|\lambda|+|L(\xi)|) \leq C(1+|\lambda+L(\xi)|) .
$$

In view of representation (3.6) and estimate (3.8), we need to show uniform $R$-boundedness of the following sets

$$
\begin{aligned}
& \left\{\xi^{\sigma}\left[D_{\xi}^{\gamma} A(\xi)+D_{\xi}^{\gamma} a_{\alpha}(\xi)\right][D(\xi, \lambda)]^{|\beta|} ; \xi \in \mathbb{R}^{n} \backslash\{0\}\right\}, \\
& \left\{\sum_{|\alpha| \leq l}|\lambda|^{1-\frac{|\alpha|}{l}} \xi^{\sigma}\left[D_{\xi}^{\gamma} A(\xi)+D_{\xi}^{\gamma} a_{\alpha}(\xi)\right][D(\xi, \lambda)]^{|\beta|} ; \xi \in \mathbb{R}^{n} \backslash\{0\}\right\}
\end{aligned}
$$

for $|\sigma|+|\gamma| \leq|\beta|$. By virtue of Kahane's contraction principle, additional and product properties of $R$-bounded operators, see, e.g., Lemma 3.5, Proposition 3.4 in [5], and in view of (3.7), it is sufficient to prove uniform $R$-boundedness of the following set

$$
B(\xi, \lambda)=\left\{Q(\xi, \lambda) ; \xi \in \mathbb{R}^{n} \backslash\{0\}\right\}, \quad Q(\xi, \lambda)=\sum_{|\alpha| \leq l}|\lambda|^{1-\frac{|\alpha|}{l}} a_{\alpha}(\xi) \xi^{\alpha} D(\xi, \lambda) .
$$

Since

$$
Q(\xi, \lambda)=\sum_{|\alpha| \leq l}|\lambda|^{1-\frac{|\alpha|}{l}} a_{\alpha}(\xi) \xi^{\alpha}[\lambda+L(\xi)]^{-1} \sigma(\xi, \lambda)
$$

thanks to $R$-boundedness of $B_{2}(\xi, \lambda)$, we have

$$
\int_{0}^{1}\left\|\sum_{j=1}^{m} r_{j}(y) \sigma\left(\eta_{j}, \lambda\right) u_{j}\right\|_{E} d y \leq C \int_{0}^{1}\left\|\sum_{j=1}^{m} r_{j}(y) u_{j}\right\|_{E} d y
$$

for all $\xi_{1}, \xi_{2}, \ldots, \xi_{m} \in \mathbb{R}^{n}, \eta_{j}=\left(\xi_{j 1}, \xi_{j 2}, \ldots, \xi_{j n}\right) \in \mathbb{R}^{n}, u_{1}, u_{2}, \ldots, u_{m} \in E, m \in N$, where $\left\{r_{j}\right\}$ is a sequence of independent symmetric $\{-1,1\}$-valued random variables on $[0,1]$. Thus, in 
view of Kahane's contraction principle, additional and product properties of $R$-bounded operators and (3.9), we obtain

$$
\begin{aligned}
\int_{0}^{1}\left\|\sum_{j=1}^{m} r_{j}(y) Q\left(\eta_{j}, \lambda\right) u_{j}\right\|_{E} d y & \leq C \int_{0}^{1}\left\|\sum_{j=1}^{m} \sigma\left(\eta_{j}, \lambda\right) r_{j}(y) u_{j}\right\|_{E} d y \\
& \leq C \int_{0}^{1}\left\|\sum_{j=1}^{m} r_{j}(y) u_{j}\right\|_{E} d y .
\end{aligned}
$$

The estimate (3.10) implies $R$-boundedness of the set $B(\xi, \lambda)$. Moreover, from Lemma 3.2, we get

$$
\sup _{\lambda} R\left\{Q(\xi, \lambda): \xi \in \mathbb{R}^{n} \backslash\{0\}\right\} \leq C
$$

i.e., we obtain the assertion.

The following result is the corollary of Lemma 3.4 and Proposition 2.4.

Result 3.5 Suppose that all conditions of Lemma 3.3 are satisfied, $E$ is UMD space, and $A(\xi)$ is a uniformly $R$-positive operator in $E$. Then the sets $S_{i}(\xi, \lambda), i=0,1,2$ are uniformly R-bounded.

Now, we are ready to present our main results. We find sufficient conditions that guarantee separability of problem (1.1).

Condition 3.6 Suppose that the following are satisfied

1. For $\varphi_{1} \in[0, \pi)$ and $\xi \in \mathbb{R}^{n}, L(\xi)=\sum_{|\alpha| \leq l} \hat{a}_{\alpha}(\xi)(i \xi)^{\alpha} \in S_{\varphi_{1}},|L(\xi)| \geq C \sum_{k=1}^{n}\left|\hat{a}_{k} \xi_{k}\right|^{l}$;

2. $\hat{a}_{\alpha} \in C^{(n)}\left(\mathbb{R}^{n}\right)$ and $|\xi|^{\beta}\left|D^{\beta} \hat{a}_{\alpha}(\xi)\right| \leq C_{1}, \beta_{k} \in\{0,1\}, 0 \leq|\beta| \leq n$;

3. For $0 \leq|\beta| \leq n$ and $\xi \in \mathbb{R}^{n} \backslash\{0\}$,

$$
\left[D^{\beta} \hat{A}(\xi)\right] \hat{A}^{-1}(\xi) \in C\left(\mathbb{R}^{n} ; B(E)\right), \quad|\xi|^{\beta}\left\|\left[D^{\beta} \hat{A}(\xi)\right] \hat{A}^{-1}(\xi)\right\|_{B(E)} \leq C_{2} .
$$

Theorem 3.7 Suppose that Condition 3.6 holds, and $E$ is a Banach space satisfying the uniform multiplier condition. Let $\hat{A}$ be a uniformly $R$-positive in $E$ with $0 \leq \varphi<\pi-\varphi_{1}$. Then, problem (1.1) has a unique solution $u$, and the following coercive uniform estimate holds

$$
\begin{aligned}
& \sum_{|\alpha| \leq l}|\lambda|^{1-\frac{|\alpha|}{l}}\left\|a_{\alpha} * D^{\alpha} u\right\|_{L_{p}\left(\mathbb{R}^{n} ; E\right)}+\|A * u\|_{L_{p}\left(\mathbb{R}^{n} ; E\right)}+|\lambda|\|u\|_{L_{p}\left(\mathbb{R}^{n} ; E\right)} \\
& \quad \leq C\|f\|_{L_{p}\left(\mathbb{R}^{n} ; E\right)}
\end{aligned}
$$

for all $f \in L_{p}\left(\mathbb{R}^{n} ; E\right), p \in(1, \infty)$ and $\lambda \in S_{\varphi}$.

Proof By applying the Fourier transform to equation (1.1), we get

$$
\hat{u}(\xi)=D(\xi, \lambda) \hat{f}(\xi), \quad D(\xi, \lambda)=[\hat{A}(\xi)+L(\xi)+\lambda]^{-1} .
$$


Hence, the solution of equation (1.1) can be represented as $u(x)=F^{-1} D(\xi, \lambda) \hat{f}$. Then there are positive constants $C_{1}$ and $C_{2}$, so that

$$
\begin{aligned}
& C_{1}|\lambda|\|u\|_{L_{p}\left(\mathbb{R}^{n} ; E\right)} \leq\left\|F^{-1}\left[\sigma_{0}(\xi, \lambda) \hat{f}\right]\right\|_{L_{p}\left(\mathbb{R}^{n} ; E\right)} \leq C_{2}|\lambda|\|u\|_{L_{p}\left(\mathbb{R}^{n} ; E\right)}, \\
& C_{1}\|A * u\|_{L_{p}\left(\mathbb{R}^{n} ; E\right)} \leq\left\|F^{-1}\left[\sigma_{1}(\xi, \lambda) \hat{f}\right]\right\|_{L_{p}\left(\mathbb{R}^{n} ; E\right)} \leq C_{2}\|A * u\|_{L_{p}\left(\mathbb{R}^{n} ; E\right)}, \\
& C_{1} \sum_{|\alpha| \leq l}|\lambda|^{1-\frac{|\alpha|}{l}}\left\|a_{\alpha} * D^{\alpha} u\right\|_{L_{p}\left(\mathbb{R}^{n} ; E\right)} \leq\left\|F^{-1}\left[\sigma_{2}(\xi, \lambda) \hat{f}\right]\right\| \|_{L_{p}\left(\mathbb{R}^{n} ; E\right)} \\
& \leq C_{2} \sum_{|\alpha| \leq l}|\lambda|^{1-\frac{|\alpha|}{l}}\left\|a_{\alpha} * D^{\alpha} u\right\|_{L_{p}\left(\mathbb{R}^{n} ; E\right)},
\end{aligned}
$$

where $\sigma_{i}(\xi, \lambda)$ are operator functions defined in Lemma 3.3. Therefore, it is sufficient to show that the operator-functions $\sigma_{i}(\xi, \lambda)$ are UBM in $L_{p}\left(\mathbb{R}^{n} ; E\right)$. However, these follow from Lemma 3.4. Thus, from (3.13), we obtain

$$
\begin{aligned}
& |\lambda|\|u\|_{L_{p}\left(\mathbb{R}^{n} ; E\right)} \leq C_{0}\|f\|_{L_{p}\left(\mathbb{R}^{n} ; E\right)}, \quad\|A * u\|_{L_{p}\left(\mathbb{R}^{n} ; E\right)} \leq C_{1}\|f\|_{L_{p}\left(\mathbb{R}^{n} ; E\right)}, \\
& \sum_{|\alpha| \leq l}|\lambda|^{1-\frac{|\alpha|}{l}}\left\|a_{\alpha} * D^{\alpha} u\right\|_{L_{p}\left(\mathbb{R}^{n} ; E\right)} \leq C_{2}\|f\|_{L_{p}\left(\mathbb{R}^{n} ; E\right)}
\end{aligned}
$$

for all $f \in L_{p}\left(\mathbb{R}^{n} ; E\right)$. Hence, we get assertion.

Let $O$ be an operator in $X=L_{p}\left(\mathbb{R}^{n} ; E\right)$ that is generated by the problem (1.1) for $\lambda=0$, i.e.,

$$
D(O) \subset W_{p}^{l}\left(\mathbb{R}^{n} ; E(A), E\right), \quad O u=\sum_{|\alpha| \leq l} a_{\alpha} * D^{\alpha}+A * u .
$$

Result 3.8 Theorem 2.6 implies that the operator $O$ is separable in $X$, i.e., for all $f \in X$, all terms of equation (1.1) also are from $X$, and for solution $u$ of equation (1.1), there are positive constants $C_{1}$ and $C_{2}$ so that

$$
C_{1}\|O u\|_{X} \leq \sum_{|\alpha| \leq l}\left\|a_{\alpha} * D^{\alpha} u\right\|_{X}+\|A * u\|_{X} \leq C_{2}\|O u\|_{X}
$$

Condition 3.9 Let $D(A)=D(\hat{A})=D\left(\hat{A}\left(\xi_{0}\right)\right)$ for $\xi_{0} \in \mathbb{R}^{n}$. Moreover, there are positive constants $C_{1}$ and $C_{2}$ so that for $u \in D(A), x \in \mathbb{R}^{n}$

$$
C_{1}\left\|\hat{A}\left(\xi_{0}\right) u\right\| \leq\|A(x) u\| \leq C_{2}\left\|\hat{A}\left(\xi_{0}\right) u\right\| .
$$

Remark 3.10 Condition 3.9 is checked for the regular elliptic operators with smooth coefficients on sufficiently smooth domains $\Omega \subset R^{m}$ considered in the Banach space $E=L_{p_{1}}(\Omega), p_{1} \in(1, \infty)$ (see Theorem 5.1).

Theorem 3.11 Assume that all conditions of Theorem 3.7 and Condition 3.9 are satisfied. Let E be a Banach space satisfying the uniform multiplier condition. Then, problem (1.1) has 
a unique solution $u \in W_{p}^{l}\left(\mathbb{R}^{n} ; E(A), E\right)$, and the following coercive uniform estimate holds

$$
\sum_{|\alpha| \leq l}|\lambda|^{1-\frac{|\alpha|}{l}}\left\|D^{\alpha} u\right\|_{L_{p}\left(\mathbb{R}^{n} ; E\right)}+\|A u\|_{L_{p}\left(\mathbb{R}^{n} ; E\right)} \leq M\|f\|_{L_{p}\left(\mathbb{R}^{n} ; E\right)}
$$

for all $f \in L_{p}\left(\mathbb{R}^{n} ; E\right), p \in(1, \infty)$ and $\lambda \in S(\varphi)$.

Proof By applying the Fourier transform to equation (1.1), we obtain $D(\xi, \lambda) \hat{u}(\xi)=\hat{f}(\xi)$, where

$$
D(\xi, \lambda)=[\hat{A}(\xi)+L(\xi)+\lambda]^{-1} .
$$

So, we obtain that the solution of equation (1.1) can be represented as $u(x)=F^{-1} D(\xi, \lambda) \hat{f}$. Moreover, by Condition 3.9, we have

$$
\left\|A F^{-1} D(\xi, \lambda) \hat{f}\right\|_{L_{p}\left(\mathbb{R}^{n} ; E\right)} \leq M\left\|\hat{A}\left(\xi_{0}\right) F^{-1} D(\xi, \lambda) \hat{f}\right\|_{L_{p}\left(\mathbb{R}^{n} ; E\right)} .
$$

Hence, by using estimates (3.12), it is sufficient to show that the operator functions $\sum_{|\alpha| \leq l}|\lambda|^{1-\frac{|\alpha|}{l}} \xi^{\alpha} D(\xi, \lambda)$ and $\hat{A}\left(\xi_{0}\right) D(\xi, \lambda)$ are UBM in $L_{p}\left(\mathbb{R}^{n} ; E\right)$. Really, in view of Condition 3.9, and uniformly $R$-positivity of $\hat{A}$, these are proved by reasoning as in Lemma 3.4.

Condition 3.12 There are positive constants $C_{1}$ and $C_{2}$ such that

$$
C_{1} \sum_{k=1}^{n}\left|a_{k} \xi_{k}\right|^{l} \leq|L(\xi)| \leq C_{2} \sum_{k=1}^{n}\left|a_{k} \xi_{k}\right|^{l}
$$

for $\xi \in \mathbb{R}^{n}$ and

$$
C_{1}\left\|A\left(x_{0}\right) u\right\| \leq\|A(x) u\| \leq C_{2}\left\|A\left(x_{0}\right) u\right\|
$$

in cases, where $D(A)=D(\hat{A})=D\left(A\left(x_{0}\right)\right), \hat{A}(\xi) A^{-1}\left(x_{0}\right) \in L_{\infty}\left(\mathbb{R}^{n} ; B(E)\right)$ for $\xi, x, x_{0} \in \mathbb{R}^{n}$ and $u \in D(A)$.

Theorem 3.13 Let all conditions of Theorem 3.11 and Condition 3.12 hold. Then for $u \in$ $W_{p}^{l}\left(\mathbb{R}^{n} ; E(A), E\right)$, there are positive constants $M_{1}$ and $M_{2}$, so that

$$
\begin{aligned}
M_{1}\|u\|_{W_{p}^{l}\left(\mathbb{R}^{n} ; E(A), E\right)} & \leq \sum_{|\alpha| \leq l}\left\|a_{\alpha} * D^{\alpha} u\right\|_{X}+\|A * u\|_{X} \\
& \leq M_{2}\|u\|_{W_{p}^{l}\left(\mathbb{R}^{n} ; E(A), E\right)} .
\end{aligned}
$$

Proof The left part of the inequality above is derived from Theorem 3.11. So, it remains to prove the right side of the estimate. Really, from Condition 3.12 for $u \in W_{p}^{l}\left(\mathbb{R}^{n} ; E(A), E\right)$ we have

$$
\|A * u\|_{X} \leq M\left\|F^{-1} \hat{A} \hat{u}\right\|_{X} \leq C\left\|F^{-1} \hat{A} A^{-1}\left(x_{0}\right) A\left(x_{0}\right) \hat{u}\right\|_{X} \leq C\left\|F^{-1} A\left(x_{0}\right) \hat{u}\right\|_{X} \leq C\|A u\|_{X} .
$$


Hence, applying the Fourier transform to equation (1.1), and by reasoning as Theorem 3.11, it is sufficient to prove that the function

$$
\sum_{|\alpha| \leq l} \hat{a}_{\alpha} \xi^{\alpha}\left[\sum_{k=1}^{n} \xi_{k}^{l_{k}}\right]^{-1}
$$

is a multiplier in $L_{p}\left(\mathbb{R}^{n} ; E\right)$. In fact, by using Condition 3.12 and the proof of Lemma 3.2, we get desired result.

Result 3.14 Theorem 3.13 implies that for all $u \in W_{p}^{l}\left(\mathbb{R}^{n} ; E(A), E\right)$, there are positive constants $C_{1}$ and $C_{2}$, so that

$$
C_{1}\|u\|_{W_{p}^{l}\left(\mathbb{R}^{n} ; E(A), E\right)} \leq\|O u\|_{L_{p}\left(\mathbb{R}^{n} ; E\right)} \leq C_{2}\|u\|_{W_{p}^{l}\left(\mathbb{R}^{n} ; E(A), E\right)}
$$

From Theorem 3.7, we have the following.

Result 3.15 Assume all conditions of Theorem 3.7 hold. Then, for all $\lambda \in S_{\varphi}$, the resolvent of operator $O$ exists, and the following sharp estimate holds

$$
\sum_{|\alpha| \leq l}|\lambda|^{1-\frac{|\alpha|}{l}}\left\|a_{\alpha} * D^{\alpha}(O+\lambda)^{-1}\right\|_{B(X)}+\left\|A *(O+\lambda)^{-1}\right\|_{B(X)}+\left\|\lambda(O+\lambda)^{-1}\right\|_{B(X)} \leq C .
$$

Result 3.16 Theorem 3.7 particularly implies that the operator $O+a$ for $a>0$ is positive in $L_{p}\left(\mathbb{R}^{n} ; E\right)$, i.e., if $\hat{A}$ is uniformly R-positive for $\varphi \in\left(\frac{\pi}{2}, \pi\right)$, then (see, e.g., [28], $\left.\$ 1.14 .5\right)$ the operator $O+a$ is a generator of an analytic semigroup in $L_{p}\left(\mathbb{R}^{n} ; E\right)$.

From Theorems 3.7, 3.11, 3.13 and Proposition 2.4, we obtain the following.

Result 3.17 Let conditions of Theorems 3.7, 3.11, 3.13 hold for Banach spaces E $\in U M D$, respectively. Then assertions of Theorems 3.7, 3.11, 3.13 are valid.

\section{The quasilinear CDOE}

Consider the equations

$$
\sum_{|\alpha|=l} a_{\alpha} * D^{\alpha} u+\left(A * D^{\sigma} u\right) u=F\left(x, D^{\sigma} u\right)+f(x), \quad x \in \mathbb{R}^{n}
$$

in $E$-valued $L_{p}$ spaces, where $A=A(x)$ is a possible unbounded operator in Banach space $E$, $a_{\alpha}=a_{\alpha}(x)$ are complex-valued functions, and $D^{\sigma}$ denote all differential operators that $|\sigma| \leq l-1$. Let

$$
\begin{aligned}
& X=L_{p}\left(\mathbb{R}^{n} ; E\right), \quad Y=W_{p}^{l}\left(\mathbb{R}^{n} ; E(A), E\right), \\
& E_{j}=(E(A), E)_{\varkappa_{\sigma}, p}, \quad \varkappa_{\sigma}=\frac{p|\sigma|+1}{p l}, \quad E_{0}=\prod_{|\sigma|<l-1} E_{\varkappa_{\sigma}} .
\end{aligned}
$$


Remark 4.1 By using Theorem 2.7, we obtain that the embedding $D^{\varkappa_{\sigma}} Y \in E_{\varkappa_{\sigma}}$ is continuous, and by trace theorem [32] (or [19]) for $w \in Y, W=\left\{w_{\varkappa_{\sigma}}\right\}, w_{\varkappa_{\sigma}}=D^{\sigma} w(\cdot),|\sigma|<l-1$,

$$
\begin{aligned}
& \prod_{|\sigma|<l-1}\left\|D^{\sigma} w\right\|_{C\left(\left(\mathbb{R}^{n}\right), E_{\varkappa \sigma}\right)}=\prod_{|\sigma|<l-1} \sup _{x \in \mathbb{R}^{n}}\left\|D^{j} w(x)\right\|_{E_{\varkappa \sigma}} \leq\|w\|_{Y}, \\
& E_{r}=\left\{v \in E_{0},\|v\|_{E_{0}} \leq r\right\}, \quad 0<r \leq r_{0} .
\end{aligned}
$$

Let $A(x, 0)$ denote by $A_{0}(x)$. Consider the linear CDOE

$$
\sum_{|\alpha|=l} a_{\alpha} * D^{\alpha} w+A_{0} * w=Q(x)
$$

From Theorem 3.7, we conclude that problem (4.2) has a unique solution $w \in W_{p}^{l}\left(\mathbb{R}^{n}\right.$; $E(A), E)$, and the coercive uniform estimate holds

$$
\sum_{|\alpha| \leq l}\left\|D^{\alpha} w\right\|_{L_{p}\left(\mathbb{R}^{n} ; E\right)}+\left\|A_{0} w\right\|_{L_{p}\left(\mathbb{R}^{n} ; E\right)} \leq M\|f\|_{L_{p}\left(\mathbb{R}^{n} ; E\right)}
$$

for all $Q \in L_{p}\left(\mathbb{R}^{n} ; E\right), p \in(1, \infty)$.

Condition 4.2 Assume that all conditions of Theorem 3.11 are satisfied for $A=A_{0}$ and $\left\|a_{\alpha}\right\|_{L_{1}}<\frac{1}{2}$. Suppose that

1. The function: $v \rightarrow A(x, v)$ is a Lipschitz function from $E_{0}$ to $B(E(A)$,E), i.e.,

$$
\|A(x, u)-A(x, v)\|_{B(E(A), E)} \leq L\|u-v\|_{E_{0}}
$$

for all $x \in \mathbb{R}^{n}$;

2. $F: \mathbb{R}^{n} \times E_{0} \rightarrow E$ is a measurable function for each $u, \bar{u} \in E_{r_{0}}, u=\left\{v_{\varkappa_{\sigma}}\right\}, \bar{u}=\left\{\bar{u}_{\varkappa_{\sigma}}\right\}$, $u_{\varkappa_{\sigma}}, \bar{u}_{\varkappa_{\sigma}} \in E_{\varkappa_{\sigma}}$, and $F(x, \cdot)$ is continuous with respect to $x \in \mathbb{R}^{n}, F(x, 0) \in X$.

Moreover, there exists $g_{i}(x)$ such that

$$
\begin{aligned}
& \|F(x, u)\|_{E} \leq g_{1}(x)\|u\|_{E_{0}}, \\
& \|F(x, u)-F(x, \bar{u})\|_{E} \leq g_{2}(x)\|u-\bar{u}\|_{E_{0}},
\end{aligned}
$$

for all $x \in \mathbb{R}^{n}, u, v \in E_{r_{0}}, g_{i} \in L_{p}\left(\mathbb{R}^{n}\right)$ and $\left\|g_{i}\right\|_{L_{p}\left(\mathbb{R}^{n}\right)} \leq M^{-1}, i=1,2$.

Theorem 4.3 Let Condition 4.2 hold. Then, there exist a radius $0<r \leq r_{0}$ and $\delta>0$ such that for each $f \in L_{p}\left(\mathbb{R}^{n}, E\right)$ with $\|f\|_{L_{p}\left(\mathbb{R}^{n}\right)} \leq \delta$ there exists a unique $u \in W_{p}^{l}\left(\mathbb{R}^{n} ; E(A), E\right)$ with $\|u\|_{W_{p}^{l}\left(\mathbb{R}^{n} ; E(A), E\right)} \leq r$ satisfying equation (3.13).

Proof We want to to solve problem (4.1) locally by means of maximal regularity of the linear problem (4.2) via the contraction mapping theorem. For this purpose, let $w$ be a solution of the linear BVP (4.2). Consider the following ball

$$
B_{r}=\left\{v \in Y,\|v\|_{Y} \leq r\right\} .
$$

Let $f \in L_{p}\left(\mathbb{R}^{n} ; E\right)$ such that $\|f\|_{L_{p}\left(\mathbb{R}^{n}\right)} \leq \delta$. Let $v \in Y,\|v\|_{Y} \leq r$. 
Define a map $G$ on $B_{r}$ by

$$
G v=u,
$$

where $u$ is a solution of problem (4.1). We want to show that $Q\left(B_{r}\right) \subset B_{r}$, and that $L$ is a contraction operator in $Y$. Consider the function

$$
Q(x)=\left(\left(A_{0}-A\right) * D^{\sigma} v\right) v+F\left(x, D^{\sigma} v\right)+f(x) .
$$

We claim that $Q \in X$, moreover, $\delta$ and $g_{i}$ can be chosen such that $M\|Q\|_{X} \leq \delta$. In fact, since by Theorem $2.7, v \in C\left(\mathbb{R}^{n} ; E_{\varkappa_{\sigma}}\right)$, and one has

$$
A(x, u)-A_{0}(x) \in C\left(\mathbb{R}^{n} ; B\left(E\left(A_{0}\right), E\right)\right) .
$$

Thus, $Q$ is measurable and

$$
\|Q\|_{E} \leq L\|v\|_{C\left(\mathbb{R}^{n} ; E_{\varkappa_{\sigma}}\right)}\|v\|_{E\left(A_{0}\right)}+g_{1}(x)\|v\|_{C\left(\mathbb{R}^{n} ; E_{\varkappa_{\sigma}}\right)}+\|f\|_{X}
$$

Now, by Remark 4.1, $\|v\|_{C\left(\mathbb{R}^{n} ; E_{\varkappa_{\sigma}}\right)} \leq\|v\|_{Y} \leq r$, by choosing $M L r+M\left\|h_{1}\right\|_{L_{p}}<\frac{1}{2}$ and $\delta=$ $r\left(\frac{1}{2} M^{-1}-L r-\left\|h_{1}\right\|_{L_{p}}\right)$, it follows that

$$
\begin{aligned}
M\|Q\|_{Y} & \leq M\left[L r\|v\|_{L_{p}\left(\mathbb{R}^{n} E\right)}+r\left\|h_{1}\right\|_{L_{p}}+\delta\right] \\
& \leq M\left[L r^{2}+r\left\|h_{1}\right\|_{L_{p}}+\delta\right]<\frac{1}{2} r .
\end{aligned}
$$

Moreover, by Theorem 3.11 and by embedding Theorem 2.6, we get

$$
\left\|\sum_{|\alpha|=l} a_{\alpha} * D^{\alpha} v\right\|_{L_{p}\left(\mathbb{R}^{n} E\right)}<\frac{1}{2} r .
$$

Thus, $G$ maps the set $B_{r}$ to $B_{r}$. Let us show that $G$ is a strict contraction. Let

$$
u_{1}=G v_{1}, \quad u_{2}=G v_{2}, \quad v_{1}, v_{2} \in B_{r}
$$

It is clearly seen that $u_{1}-u_{2}$ is a solution of the linear problem (4.2) for

$$
Q=\left(\left(A_{0}-A\right) * D^{\sigma} v\right) v+F\left(x, D^{\sigma} v\right) .
$$

Then, by using estimate (4.3) and reasoning as above, we get

$$
\begin{aligned}
\left\|u_{1}-u_{2}\right\|_{Y} & \leq M\|Q\|_{X} \\
& \leq M\left\{L r\left\|v_{1}-v_{2}\right\|_{X}+L\left\|v_{1}-v_{2}\right\|_{Y}\left\|v_{1}\right\|_{L_{p}\left(\mathbb{R}^{n} ; E\left(A_{0}\right)\right)}\left\|h_{2}\right\|_{L_{p}}\left\|v_{1}-v_{2}\right\|_{Y}\right\} \\
& \leq M\left(2 L r+\left\|h_{2}\right\|_{L_{p}}\right)\left\|v_{1}-v_{2}\right\|_{Y} .
\end{aligned}
$$

Choose $h_{2}$, so that $\left\|h_{2}\right\|_{L_{p}}<\frac{1}{M}-2 L r$, we obtain that $G$ is a strict contraction. Then by virtue of contraction mapping principle, we obtain that problem (4.1) has a unique solution $u \in W_{p}^{l}\left(\mathbb{R}^{n} ; E(A), E\right)$. 


\section{Boundary value problems for integro-differential equations}

In this section, by applying Theorem 3.7, the BVP for the anisotropic type convolution equations is studied. The maximal regularity of this problem in mixed $L_{\mathbf{p}}$ norms is derived. In this direction, we can mention, e.g., the works [2, 18, 21] and [33].

Let $\tilde{\Omega}=\mathbb{R}^{n} \times \Omega$, where $\Omega \subset R^{\mu}$ is an open connected set with a compact $C^{2 m}$-boundary $\partial \Omega$. Consider the BVP for integro-differential equation

$$
\begin{aligned}
& (L+\lambda) u=\sum_{|\alpha| \leq l} a_{\alpha} * D^{\alpha} u+\sum_{|\alpha| \leq 2 m}\left(b_{\alpha} \eta_{\alpha} D_{y}^{\alpha}+\lambda\right) * u=f(x, y), \quad x \in \mathbb{R}^{n}, y \in \Omega, \\
& B_{j} u=\sum_{|\beta| \leq m_{j}} b_{j \beta}(y) D_{y}^{\beta} u(x, y)=0, \quad y \in \partial \Omega, j=1,2, \ldots, m,
\end{aligned}
$$

where

$$
\begin{aligned}
& D_{j}=-i \frac{\partial}{\partial y_{j}}, \quad y=\left(y_{1}, \ldots, y_{\mu}\right), \quad b_{\alpha}=b_{\alpha}(x), \quad \eta_{\alpha}=\eta_{\alpha}(y), \\
& a_{\alpha}=a_{\alpha}(x), \quad \alpha=\left(\alpha_{1}, \alpha_{2}, \ldots, \alpha_{n}\right), \quad a_{\alpha}=a_{\alpha}(x), \quad u=u(x, y) .
\end{aligned}
$$

In general, $l \neq 2 m$, so equation (4.4) is anisotropic. For $l=2 m$, we get isotropic equation. If $\tilde{\Omega}=\mathbb{R}^{n} \times \Omega, \mathbf{p}=\left(p_{1}, p\right), L_{\mathbf{p}}(\tilde{\Omega})$ will denote the space of all $\mathbf{p}$-summable scalar-valued functions with a mixed norm (see, e.g., [34]), i.e., the space of all measurable functions $f$ defined on $\tilde{\Omega}$, for which

$$
\|f\|_{L_{\mathbf{p}}(\tilde{\Omega})}=\left(\int_{\mathbb{R}^{n}}\left(\int_{\Omega}|f(x, y)|^{p_{1}} d x\right)^{\frac{p}{p_{1}}} d y\right)^{\frac{1}{p}}<\infty
$$

Analogously, $W_{\mathbf{p}}^{l}(\tilde{\Omega})$ denotes the Sobolev space with a corresponding mixed norm [34]. Let $Q$ denote the operator, generated by problem (4.4) and (5.1). In this section, we present the following result.

\section{Theorem 5.1 Let the following conditions be satisfied}

1. $\eta_{\alpha} \in C(\bar{\Omega})$ for each $|\alpha|=2 m$ and $\eta_{\alpha} \in L_{\infty}(\Omega)+L_{r_{k}}(\Omega)$ for each $|\alpha|=k<2 m$ with $r_{k} \geq p_{1}, p_{1} \in(1, \infty)$ and $2 m-k>\frac{l}{r_{k}}, v_{\alpha} \in L_{\infty}$;

2. $b_{j \beta} \in C^{2 m-m_{j}}(\partial \Omega)$ for each $j, \beta, m_{j}<2 m, p \in(1, \infty), \lambda \in S_{\varphi}, \varphi \in[0, \pi)$;

3. For $y \in \bar{\Omega}, \xi \in R^{\mu}, \sigma \in S_{\varphi_{0}}, \varphi_{0} \in\left(0, \frac{\pi}{2}\right),|\xi|+|\sigma| \neq 0$ let $\sigma+\sum_{|\alpha|=2 m} \eta_{\alpha}(y) \xi^{\alpha} \neq 0$;

4. For each $y_{0} \in \partial \Omega$ local BVP in local coordinates corresponding to $y_{0}$

$$
\begin{aligned}
& \sigma+\sum_{|\alpha|=2 m} \eta_{\alpha}\left(y_{0}\right) D^{\alpha} \vartheta(y)=0, \\
& B_{j 0} \vartheta=\sum_{|\beta|=m_{j}} b_{j \beta}\left(y_{0}\right) D^{\beta} \vartheta(y)=h_{j}, \quad j=1,2, \ldots, m
\end{aligned}
$$

has a unique solution $\vartheta \in C_{0}\left(R_{+}\right)$for all $h=\left(h_{1}, h_{2}, \ldots, h_{m}\right) \in R^{m}$ and for $\xi^{\prime} \in R^{\mu-1}$ with $\left|\xi^{\prime}\right|+|\lambda| \neq 0$; 
5. The (1) part of Condition 3.6 is satisfied, $\hat{a}_{\alpha}, \hat{b}_{\alpha} \in C^{(n)}\left(\mathbb{R}^{n}\right)$, and there are positive constants $C_{i}, i=1,2$, so that

$$
\begin{aligned}
& |\xi|^{\beta}\left|D^{\beta} \hat{a}_{\alpha}(\xi)\right| \leq C_{1}, \quad|\xi|^{\beta}\left|D^{\beta} \hat{b}_{\alpha}(\xi)\right| \leq C_{2}\left|\hat{b}_{\alpha}(\xi)\right|, \\
& \xi \in \mathbb{R}^{n} \backslash\{0\}, \quad \beta_{k} \in\{0,1\}, \quad 0 \leq|\beta| \leq n .
\end{aligned}
$$

Then, for $f \in W_{\mathbf{p}}^{l}(\tilde{\Omega})$ and $\lambda \in S_{\varphi}$ problems (4.4) and (5.1) have a unique solution $u \in W_{p}^{l}(\tilde{\Omega})$, and the following coercive uniform estimate holds

$$
\sum_{|\alpha| \leq l}|\lambda|^{1-\frac{|\alpha|}{l}}\left\|a_{\alpha} * D^{\alpha} u\right\|_{L_{\mathbf{p}}(\tilde{\Omega})}+\||\lambda| u\|_{L_{\mathbf{p}}(\tilde{\Omega})}+\sum_{|\alpha| \leq 2 m}\left\|b_{\alpha} \eta_{\alpha} D^{\alpha} * u\right\|_{L_{\mathbf{p}}(\tilde{\Omega})} \leq C\|f\|_{L_{\mathbf{p}}(\tilde{\Omega})} .
$$

Proof Let $E=L_{p_{1}}(\Omega)$. It is known [29] that $L_{p_{1}}(\Omega)$ is $U M D$ space for $p_{1} \in(1, \infty)$. Consider the operator $A$ in $L_{p_{1}}(\Omega)$, defined by

$$
D(A)=W_{p_{1}}^{2 m}\left(\Omega ; B_{j} u=0\right), \quad A(x) u=\sum_{|\alpha| \leq 2 m} b_{\alpha}(x) \eta_{\alpha}(y) D^{\alpha} u(y) .
$$

Therefore, problems (4.4) and (5.1) can be rewritten in the form of (1.1), where $u(x)=$ $u(x, \cdot), f(x)=f(x, \cdot)$ are functions with values in $E=L_{p_{1}}(\Omega)$. It is easy to see that $\hat{A}(\xi)$ and $D^{\beta} \hat{A}(\xi)$ are operators in $L_{p_{1}}(\Omega)$ defined by

$$
\begin{aligned}
& D(\hat{A})=D\left(D^{\beta} \hat{A}\right)=W_{p_{1}}^{2 m}\left(\Omega ; B_{j} u=0\right), \quad \hat{A}(\xi) u=\sum_{|\alpha| \leq 2 m} \hat{b}_{\alpha}(\xi) \eta_{\alpha}(y) D^{\alpha} u(y), \\
& D_{\xi}^{\beta} \hat{A}(\xi) u=\sum_{|\alpha| \leq 2 m} D_{\xi}^{\beta} \hat{b}_{\alpha}(\xi) \eta_{\alpha}(y) D^{\alpha} u(y) .
\end{aligned}
$$

In view of conditions and by [5, Theorem 8.2] operators $\hat{A}(\xi)+\mu$ and $D^{\beta} \hat{A}(\xi)+\mu$ for sufficiently large $\mu>0$, are uniformly $R$-positive in $L_{p_{1}}(\Omega)$. Moreover, by (3.3), the problems

$$
\begin{aligned}
& \mu u(y)+\sum_{|\alpha| \leq 2 m} \hat{b}_{\alpha}(\xi) \eta_{\alpha}(y) D^{\alpha} u(y)=f(y), \\
& B_{j} u=\sum_{|\beta| \leq m_{j}} b_{j \beta}(y) D^{\beta} u(y)=0, \quad j=1,2, \ldots, m, \\
& \mu u(y)+\sum_{\alpha \leq 2 m} D^{\beta} \hat{b}_{\alpha}(\xi) \eta_{\alpha}(y) D^{\alpha} u(y)=f(y), \\
& B_{j} u=\sum_{|\beta| \leq m_{j}} b_{j \beta}(y) D^{\beta} u(y)=0, \quad j=1,2, \ldots, m
\end{aligned}
$$

for $f \in L_{p_{1}}(\Omega)$ and for sufficiently large $\mu$, have unique solutions that belong to $W_{p_{1}}^{l}(\Omega)$, and the coercive estimates hold

$$
\|u\|_{W_{p_{1}}^{l}(\Omega)} \leq C\|(\hat{A}+\mu) u\|_{L_{p_{1}}(\Omega)}, \quad\|u\|_{W_{p_{1}}^{2 m}(\Omega)} \leq C\left\|\left(D^{\beta} \hat{A}+\mu\right) u\right\|_{L_{p_{1}}(\Omega)}
$$


for solutions of problems (5.4) and (5.5). Then in view of (5) condition and by virtue of embedding theorems [34], we obtain

$$
\begin{aligned}
& \|(\hat{A}+\mu) u\|_{L_{p_{1}}(\Omega)} \leq C\|u\|_{W_{p_{1}}^{2 m}(\Omega)} \leq C\|(\hat{A}+\mu) u\|_{L_{p_{1}}(\Omega)}, \\
& \left\|\left(D^{\beta} \hat{A}+\mu\right) u\right\|_{L_{p_{1}}(\Omega)} \leq C\|u\|_{W_{p_{1}}^{2 m}(\Omega)} \leq C\left\|\left(D^{\beta} \hat{A}+\mu\right) u\right\|_{L_{p_{1}}(\Omega)} .
\end{aligned}
$$

Moreover by using (5) condition for $u \in W_{p_{1}}^{2 m}(\Omega)$ we have

$$
|\xi|^{\beta}\left\|\left(D_{\xi}^{\beta} \hat{A}+\mu\right) u\right\|_{L_{p_{1}(\Omega)}} \leq C\|(\hat{A}+\mu) u\|_{L_{p_{1}}(\Omega)}
$$

i.e., all conditions of Theorem 3.7 hold, and we obtain the assertion.

\section{Infinite system of IDEs}

Consider the following infinity system of a convolution equation

$$
\sum_{|\alpha| \leq l} a_{\alpha} * D^{\alpha} u_{m}+\sum_{j=1}^{\infty}\left(d_{j}+\lambda\right) * u_{j}(x)=f_{m}(x)
$$

for $x \in \mathbb{R}^{n}$ and $m=1,2, \ldots$

Condition 6.1 There are positive constants $C_{1}$ and $C_{2}$, so that for $\left\{d_{j}(x)\right\}_{1}^{\infty} \in l_{q}$ for all $x \in \mathbb{R}^{n}$ and some $x_{0} \in \mathbb{R}^{n}$,

$$
C_{1}\left|d_{j}\left(x_{0}\right)\right| \leq\left|d_{j}(x)\right| \leq C_{2}\left|d_{j}\left(x_{0}\right)\right| .
$$

Suppose that $\hat{a}_{\alpha}, \hat{d}_{m} \in C^{(n)}\left(\mathbb{R}^{n}\right)$, and there are positive constants $M_{i}, i=1,2$, so that

$$
\begin{aligned}
& |\xi|^{\beta}\left|D^{\beta} \hat{a}_{\alpha}(\xi)\right| \leq M_{1}, \quad|\xi|^{\beta}\left|D^{\beta} \hat{d}_{m}(\xi)\right| \leq M_{2}\left|\hat{d}_{m}(\xi)\right|, \\
& \xi \in \mathbb{R}^{n} \backslash\{0\}, \quad \beta_{k} \in\{0,1\}, \quad 0 \leq|\beta| \leq n .
\end{aligned}
$$

Let

$$
\begin{aligned}
& D(x)=\left\{d_{m}(x)\right\}, \quad d_{m}>0, \quad u=\left\{u_{m}\right\}, \quad D * u=\left\{d_{m} * u_{m}\right\}, \\
& l_{q}(D)=\left\{u \in l_{q},\|u\|_{l_{q}(D)}=\left(\sum_{m=1}^{\infty}\left|d_{m}\left(x_{0}\right) * u_{m}\right|^{q}\right)^{\frac{1}{q}}<\infty\right\}, \quad 1<q<\infty .
\end{aligned}
$$

Let $Q$ be a differential operator in $L_{p}\left(\mathbb{R}^{n} ; l_{q}\right)$, generated by problem $(5.7)$ and $B=$ $B\left(L_{p}\left(\mathbb{R}^{n} ; l_{q}\right)\right)$. Applying Theorem 3.7 , we have the following.

Theorem 6.2 Suppose that (1) part of Condition 3.6 and Condition 6.1 are satisfied. Then

1. For all $f(x)=\left\{f_{m}(x)\right\}_{1}^{\infty} \in L_{p}\left(\mathbb{R}^{n} ; l_{q}(D)\right)$, for $\lambda \in S_{\varphi}, \varphi \in[0, \pi)$ the equation (6.1) has a unique solution $u=\left\{u_{m}(x)\right\}_{1}^{\infty}$ that belongs to $W_{p}^{l}\left(\mathbb{R}^{n} ; l_{q}(D), l_{q}\right)$, and the coercive uniform estimate holds

$$
\sum_{|\alpha| \leq l}|\lambda|^{1-\frac{|\alpha|}{l}}\left\|a_{\alpha} * D^{\alpha} u\right\|_{L_{p}\left(\mathbb{R}^{n} ; l_{q}\right)}+\|D * u\|_{L_{p}\left(\mathbb{R}^{n} ; l_{q}\right)}+|\lambda|\|u\|_{L_{p}\left(\mathbb{R}^{n} ; l_{q}\right)} \leq C\|f\|_{L_{p}\left(\mathbb{R}^{n} ; l_{q}\right)} ;
$$


2. For $\lambda \in S_{\varphi}$, there exists a resolvent $(Q+\lambda)^{-1}$ of operator $Q$ and

$$
\sum_{|\alpha| \leq l}|\lambda|^{1-\frac{|\alpha|}{l}}\left\|a_{\alpha} *\left[D^{\alpha}(Q+\lambda)^{-1}\right]\right\|_{B}+\left\|D *(Q+\lambda)^{-1}\right\|_{B}+\left\|\lambda(Q+\lambda)^{-1}\right\|_{B} \leq C .
$$

Proof Really, let $E=l_{q}$ and $A=\left[d_{m}(x) \delta_{j m}\right], m, j=1,2, \ldots$ Then

$$
\hat{A}(\xi)=\left[\hat{d}_{m}(\xi) \delta_{j m}\right], \quad D^{\beta} \hat{A}(\xi)=\left[D^{\beta} \hat{d}_{m}(\xi) \delta_{j m}\right], \quad m, j=1,2, \ldots
$$

It is easy to see that $\hat{A}(\xi)$ is uniformly $R$-positive in $l_{q}$, and all conditions of Theorem 3.7 are hold. Therefore, by virtue of Theorem 3.7 and Result 4.1, we obtain the assertions.

Remark 6.3 There are a lot of positive operators in concrete Banach spaces. Therefore, putting concrete Banach spaces instead of $E$ and concrete positive differential, pseudo differential operators, or finite, infinite matrices, etc. instead of operator $A$ in (1.1) and (4.1), we can obtain the maximal regularity of different class of convolution equations, Cauchy problems for parabolic CDEs or it's systems, by virtue of Theorem 3.7 and Theorem 3.11, respectively.

Competing interests

The authors declare that they have no competing interests.

Authors' contributions

All authors read and approved the final manuscript.

\section{Author details}

${ }^{1}$ Department of Mechanical Engineering, Okan University, Tuzla, Istanbul, Turkey. ${ }^{2}$ Institute of Mathematics and Mechanics, Azerbaijan National Academy of Sciences, Baku, Azerbaijan. ${ }^{3}$ Department of Mathematics, Dumlupınar University, Kütahya, Turkey.

\section{Acknowledgements}

The authors would like to thank the referees for valuable comments and suggestions in improving this paper.

Received: 16 May 2013 Accepted: 31 July 2013 Published: 19 September 2013

\section{References}

1. Amann, H: Linear and Quasi-Linear Equations, vol. 1. Birkhäuser, Basel (1995)

2. Amann, H: Operator-valued Fourier multipliers, vector-valued Besov spaces, and applications. Math. Nachr. 186, 5-56 (1997)

3. Agarwal, R, Bohner, P, Shakhmurov, R: Maximal regular boundary value problems in Banach-valued weighted spaces. Bound. Value Probl. 1, 9-42 (2005)

4. Dore, G, Yakubov, S: Semigroup estimates and non coercive boundary value problems. Semigroup Forum 60, 93-121 (2000)

5. Denk, R, Hieber, M: R-boundedness, Fourier multipliers and problems of elliptic and parabolic type. Mem. Am. Math. Soc. $166,788(2003)$

6. Gorbachuk, VI, Gorbachuk, ML: Boundary Value Problems for Differential-Operator Equations. Naukova Dumka, Kiev (1984)

7. Haller, R, Heck, H, Noll, A: Mikhlin's theorem for operator-valued Fourier multipliers in $n$ variables. Math. Nachr. 244, 110-130 (2002)

8. Lunardi, A: Analytic Semigroups and Optimal Regularity in Parabolic Problems. Birkhäuser, Basel (2003)

9. Ragusa, MA: Homogeneous Herz spaces and regularity results. Nonlinear Anal., Theory Methods Appl. 71(12), e1909-e1914 (2009)

10. Sobolevskii, PE: Inequalities coerciveness for abstract parabolic equations. Dokl. Akad. Nauk SSSR 57(1), 27-40 (1964)

11. Shakhmurov, VB: Embedding and maximal regular differential operators in Banach-valued weighted spaces. Acta Math. Sin. 22(5), 1493-1508 (2006)

12. Weis, L: Operator-valued Fourier multiplier theorems and maximal $L_{p}$ regularity. Math. Ann. 319, 735-758 (2001)

13. Yakubov, S, Yakubov, Y: Differential-Operator Equations. Ordinary and Partial Differential Equations. Chapman \& Hall/CRC, Boca Raton (2000) 
14. Shakhmurov, VB: Embedding theorems and maximal regular differential operator equations in Banach-valued function spaces. J. Inequal. Appl. 4, 605-620 (2005)

15. Shakhmurov, VB: Embedding and separable differential operators in Sobolev-Lions type spaces. Mathematical Notes 84(6), 906-926 (2008)

16. Guliyev, VS: Embeding theorems for spaces of UMD-valued functions. Dokl. Akad. Nauk SSSR 329(4), 408-410 (1993) (in Russian)

17. Guliyev, VS: On the theory of multipliers of Fourier integrals for Banach spaces valued functions. In: Investigations in the Theory of Differentiable Functions of Many Variables and Its Applications. Tr. Math. Inst. Steklova, vol. 214 (1997) (in Russian)

18. Engler, $\mathrm{H}$ : Strong solutions of quasilinear integro-differential equations with singular kernels in several space dimension. Electron. J. Differ. Equ. 1995(02), 1-16 (1995)

19. Keyantuo, V, Lizama, C: Maximal regularity for a class of integro-differential equations with infinite delay in Banach spaces. Stud. Math. 168, 25-50 (2005)

20. Prüss, J: Evolutionary Integral Equations and Applications. Birkhäuser, Basel (1993)

21. Poblete, V: Solutions of second-order integro-differential equations on periodic Besov spaces. Proc. Edinb. Math. Soc. 50, 477-492 (2007)

22. Vergara, V: Maximal regularity and global well-posedness for a phase field system with memory. J. Integral Equ. Appl. 19, 93-115 (2007)

23. Shakhmurov, VB: Coercive boundary value problems for regular degenerate differential-operator equations. J. Math. Anal. Appl. 292(2), 605-620 (2004)

24. Arendt, W, Bu, S: Tools for maximal regularity. Math. Proc. Camb. Philos. Soc. 134, 317-336 (2003)

25. Girardi, $M$, Weis, L: Operator-valued multiplier theorems on $L_{p}(X)$ and geometry of Banach spaces. J. Funct. Anal. 204(2), 320-354 (2003)

26. Hytönen, T, Weis, L: Singular convolution integrals with operator-valued kernels. Math. Z. 255, $393-425$ (2007)

27. Shakhmurov, VB, Shahmurov, R: Sectorial operators with convolution term. Math. Inequal. Appl. 13(2), 387-404 (2010)

28. Triebel, H: Interpolation Theory, Function Spaces, Differential Operators. North-Holland, Amsterdam (1978)

29. Burkholder, DL: A geometrical conditions that implies the existence certain singular integral of Banach space-valued functions. In: Proc. Conf. Harmonic Analysis in Honor of Antonu Zigmund, Chicago, 1981, pp. 270-286. Wadsworth, Belmont (1983)

30. Bourgain, J: Some remarks on Banach spaces in which martingale differences are unconditional. Ark. Mat. 21, 163-168 (1983)

31. Shakhmurov, VB: Maximal B-regular boundary value problems with parameters. J. Math. Anal. Appl. 320, 1-19 (2006)

32. Lions, JL, Peetre, J: Sur une classe d'espaces d'interpolation. Publ. Math. Inst. Hautes Études Sci. 19, 5-68 (1964)

33. Shakhmurov, VB, Shahmurov, R: Maximal B-regular integro-differential equations. Chin. Ann. Math., Ser. B 30(1), 39-50 (2009)

34. Besov, OV, Ilin, VP, Nikolskii, SM: Integrals Representations of Functions and Embedding Theorem. Nauka, Moscow (1975)

doi:10.1186/1687-2770-2013-211

Cite this article as: Shakhmurov and Ekincioglu: Linear and nonlinear convolution elliptic equations. Boundary Value Problems 2013 2013:211.

\section{Submit your manuscript to a SpringerOpen ${ }^{\circ}$ journal and benefit from:}

- Convenient online submission

Rigorous peer review

Immediate publication on acceptance

- Open access: articles freely available online

- High visibility within the field

- Retaining the copyright to your article 